Vietnam Journal of Mechanics, VAST, Vol. 27, No. 3 (2005), pp. 171-178

\title{
COMPLETE SHAKING FORCE AND SHAKING MOMENT BALANCING OF SPATIAL MULTIBODY SYSTEMS WITH OPEN KINEMATIC CHAINS
}

\author{
Nguyen Van Khang, Nguyen Phong Dien, Pham Van Son \\ Hanoi University of Technology
}

\begin{abstract}
This paper deals with a solution for the problem of full shaking force and shaking moment balancing of spatial multibody systems with open kinematic chains. Firstly, the general conditions for complete dynamic balancing of spatial multibody systems are formulated. These include formulae for complete elimination of the resultant inertia force and inertia couple caused by all moving bodies. In the following example, the equations of complete shaking force and shaking moment balancing of a nine-bar direct drive manipulator are given.
\end{abstract}

\section{INTRODUCTION}

The balancing of machinery has became an important research topic because a balanced machine leads to better dynamic characteristics and less vibrations. A considerable amount of research on the dynamic balancing of planar mechanisms has been carried out for several decades. It is well known that the resultant inertia force (called as shaking force) and the resultant inertia couple (or shaking moment) caused by all moving links can be reduced by either internal mass redistribution, or using counterweights [5, 7] or adding supplementary links such as cams, dyads to the initial mechanism [6].

In contrast to the rapid progress in balancing theory of planar mechanisms, the development on the balancing theory of spatial multibody systems is still limited. Balancing methods of planar mechanisms can not be directly applicable to spatial multibody systems since kinematic and dynamic properties of spatial multibody systems are much more complicated. The literature on this respect therefore is very little (see [1]-[4]).

Based on theory of multibody dynamics, the general conditions for complete shaking force and shaking moment balancing of spatial multibody systems have been derived theoretically in this paper. These include formulae for complete elimination of the resultant inertia force and inertia couple caused by all moving links. A first attempt is made here to present a solution for the problem of complete balancing of spatial multibody systems with open kinematic chains. In the following example, the equations of shaking force and shaking moment balancing of a nine-link direct drive manipulator are given. A specialized code has been developed on the MAPLE environment for this study.

\section{GENERAL CONDITIONS FOR COMPLETE SHAKING FORCE AND SHAKING MOMENT BALANCING OF SPATIAL MULTIBODY SYSTEMS}

We consider a spatial multibody system with holonomic and rheonomic constraints as a set of linked rigid bodies in an open or a closed loop structure shown in Fig. 1. The 
shaking force $\boldsymbol{F}^{*}$ and the shaking moment $\boldsymbol{M}_{O}^{*}$ that caused by all moving bodies can be expressed in the compact matrix form [1], [8]:

$$
\begin{aligned}
& \boldsymbol{F}^{*}=-\frac{d}{d t} \boldsymbol{p}=-\frac{d}{d t} \sum_{i=1}^{n} m_{i} \boldsymbol{v}_{i}, \\
& \boldsymbol{M}_{O}^{*}=-\frac{d}{d t} \boldsymbol{L}_{O}=-\frac{d}{d t} \sum_{i=1}^{n}\left(\boldsymbol{I}_{i} \boldsymbol{\omega}_{i}+\tilde{\boldsymbol{r}}_{i} m_{i} \boldsymbol{v}_{i}\right),
\end{aligned}
$$

where $m_{i}$ is the mass of the $i$-th body, $n$ - the number of bodies, $\mathbf{r}_{i}$ - the position vector of the center of mass $C_{i}, \boldsymbol{v}_{i}$ - the velocity of the center of mass $C_{i}, \boldsymbol{\omega}_{i}$ - the angular velocity of the $i$-th body, $\mathbf{I}_{i}$ - the matrix of the inertia tensor of the $i$-th body referred to $C_{i}, \mathbf{p}$ - the linear momentum of the system, $\mathbf{L}_{O}$ - the angular momentum of the system taken about fixed point.

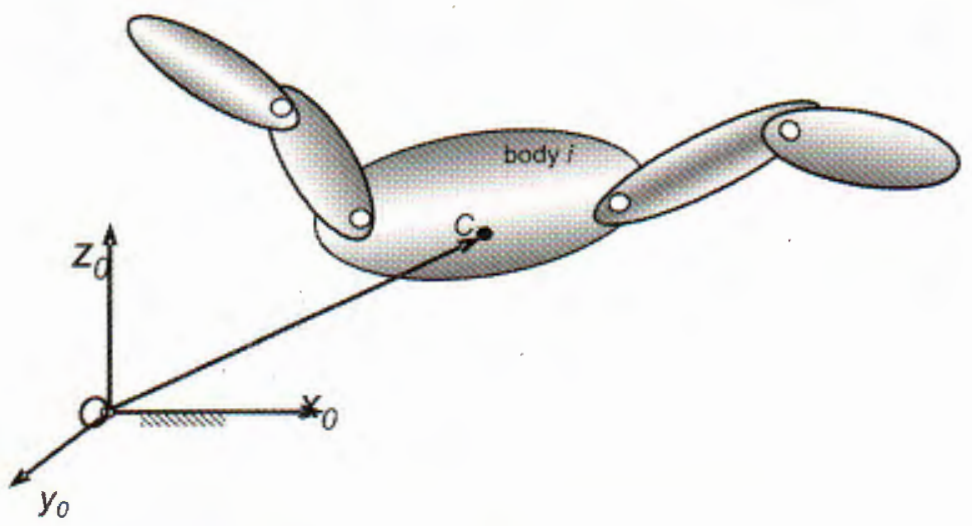

Fig. 1. A spatial multibody system

Now we choose $f$ independent generalized coordinates $\boldsymbol{q}=\left[q_{1}, q_{2}, \ldots, q_{f}\right]^{T}$ corresponding to the $f$ degrees of freedom of the system. Assumed that the system has holonomic constraints, in this case the velocity $\boldsymbol{v}_{i}$ and the angular velocity $\boldsymbol{\omega}_{i}$ are given by

$$
\begin{aligned}
\boldsymbol{v}_{i} & =\boldsymbol{J}_{T i}(\boldsymbol{q}) \dot{\boldsymbol{q}}, \\
\boldsymbol{\omega}_{i} & =J_{R i}(\boldsymbol{q}) \dot{\boldsymbol{q}},
\end{aligned}
$$

where $\boldsymbol{J}_{T i}(\omega q)$ and $\boldsymbol{J}_{R i}(\omega q)$ denote the $3 \times f$ Jacobian matrices that relate velocity $\boldsymbol{v}_{i}$ and angular velocity $\boldsymbol{\omega}_{i}$ to the generalized velocities $\dot{\boldsymbol{q}}=\left[\dot{q}_{1}, \dot{q}_{2}, \ldots, \dot{q}_{f}\right]^{T}$.

$$
\boldsymbol{J}_{T i}(q)=\frac{\partial r_{i}}{\partial \boldsymbol{q}}, \quad \boldsymbol{J}_{R i}(q)=\frac{\partial \boldsymbol{\omega}_{i}}{\partial \dot{\boldsymbol{q}}} .
$$

Substituting (2.3) and (2.4) into equations (2.1) and (2.2), the shaking force $F^{*}$ and the shaking moment $\boldsymbol{M}_{O}^{*}$ can be written in the form

$$
\begin{aligned}
& \boldsymbol{F}^{*}=-\frac{d}{d t}\left\{\left[\sum_{i=1}^{n} m_{i} \boldsymbol{J}_{T i}(\boldsymbol{q})\right] \dot{\boldsymbol{q}}\right\}, \\
& \boldsymbol{M}_{O}^{*}=-\frac{d}{d t}\left\{\left[\sum_{i=1}^{n} \boldsymbol{I}_{i} \boldsymbol{J}_{R i}(\boldsymbol{q})+m_{i} \tilde{r}_{i} \boldsymbol{J}_{T i}(\boldsymbol{q})\right] \dot{\boldsymbol{q}}\right\} .
\end{aligned}
$$


So, the spatial multibody system can be completely balanced if the shaking force and the shaking moment vanish, this yields

$$
\begin{aligned}
& \sum_{i=1}^{n} m_{i} \boldsymbol{J}_{T i}(\boldsymbol{q})=0, \\
& \sum_{i=1}^{n}\left[\boldsymbol{I}_{i} \boldsymbol{J}_{R i}(\boldsymbol{q})+m_{i} \tilde{\boldsymbol{r}}_{i} \boldsymbol{J}_{T i}(\boldsymbol{q})\right]=0 .
\end{aligned}
$$

Equations (2.8) and (2.9) are the general conditions for complete dynamic balancing of spatial multibody systems. If the mass distribution of the bodies that satisfies equation (2.8), the shaking force will be fully balanced (static balancing). However, in general, the shaking moment can not be fully balanced by internal mass redistribution [4]. One of current trend in dynamic balancing is minimizing the shaking moment of the full force balanced system [5].

Note that equations (2.8) and (2.9) are expressed in the inertial reference system. The elements of inertia matrix $\mathbf{I}_{i}$ are time dependent. The term $\boldsymbol{I}_{i} \boldsymbol{J}_{R i}(\boldsymbol{q})$ of equation (2.9) can be developed most conveniently in the body-fixed coordinate frame because the inertia components of the body are constant in this coordinate frame.

The inertia matrix $\mathbf{I}_{i}$ can be expressed as [8]

$$
\boldsymbol{I}_{i}=\boldsymbol{A}_{i} \boldsymbol{I}_{i}^{(i)} \boldsymbol{A}_{i}^{T}
$$

where $\boldsymbol{A}_{i}$ denotes the rotation matrix of the $i$-th body referred to the fixed coordinate frame, $\boldsymbol{I}_{i}^{(i)}$ is the inertia matrix determined in the body-fixed coordinate frame. Let $\boldsymbol{\omega}_{i}^{(i)}$ be the angular velocity of the $i$-th body with respect to the body-fixed coordinate frame, it can be shown that

$$
\boldsymbol{J}_{R i}(\boldsymbol{q})=\frac{\partial \boldsymbol{\omega}_{i}}{\partial \dot{\boldsymbol{q}}}=\frac{\partial\left(\boldsymbol{A}_{i} \boldsymbol{\omega}_{i}^{(i)}\right)}{\partial \dot{\boldsymbol{q}}}=\boldsymbol{A}_{i} \frac{\partial \boldsymbol{\omega}_{i}^{(i)}}{\partial \dot{\boldsymbol{q}}} .
$$

Substituting equations (2.10) and (2.11) into expression $I_{i} J_{R i}(q)$, we obtain

where

$$
\begin{gathered}
\boldsymbol{I}_{i} \boldsymbol{J}_{R i}(\boldsymbol{q})=\boldsymbol{A}_{i} \boldsymbol{I}_{i}^{(i)} \boldsymbol{A}_{i}^{T} \boldsymbol{A}_{i} \frac{\partial \boldsymbol{\omega}_{i}^{(i)}}{\partial \dot{\boldsymbol{q}}}=\boldsymbol{A}_{i} \boldsymbol{I}_{i}^{(i)} \boldsymbol{J}_{R i}^{(i)} \\
\boldsymbol{J}_{i}^{(i)}=\frac{\partial \boldsymbol{\omega}_{i}^{(i)}}{\partial \dot{\boldsymbol{q}}}
\end{gathered}
$$

Substituting (2.12) into equation (2.9), the conditions for shaking moment balancing take the form

$$
\sum_{i=1}^{n}\left[\boldsymbol{A}_{i} \boldsymbol{I}_{i}^{(i)} \boldsymbol{J}_{R i}^{(i)}(\boldsymbol{q})+m_{i} \tilde{\boldsymbol{r}}_{i} \boldsymbol{J}_{T i}(\boldsymbol{q})\right]=\mathbf{0}
$$

\section{EXAMPLE}

In the following example we introduce the application of the general balancing conditions described above to a spatial multibody system with open kinematic chains. Fig. 2 shows a nine-link direct drive manipulator designed by Abdel-Rahman and Elbestawi [2]. 


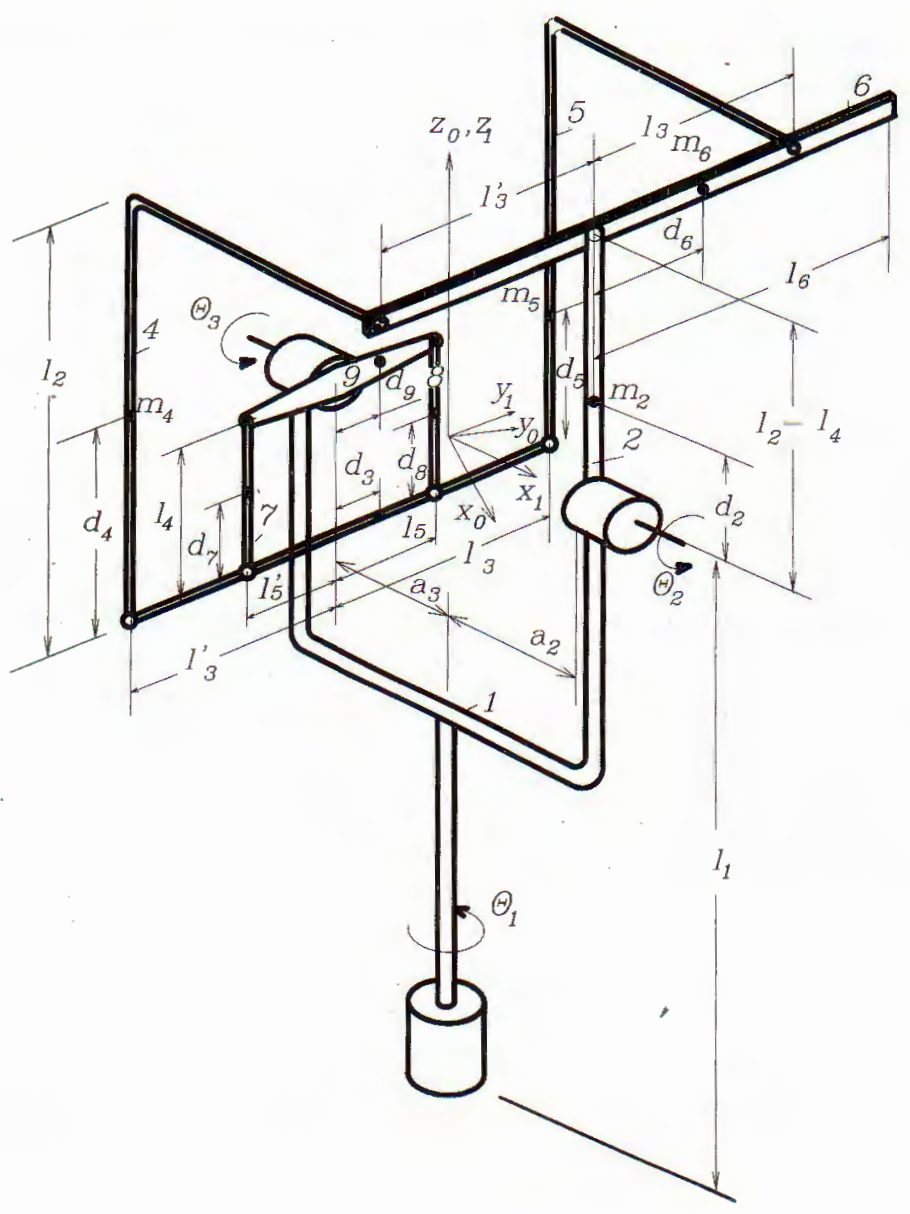

Fig. 2. A nine-link direct drive manipulator

This manipulator is connected directly to three high torque actuators. The first actuator drives link 1, the second actuator drives link 2, which drives links 4, 5, 7 and 8 consecutively. The third actuator drives link 9 and consequently, links 3 and the endeffector link 6. Link 1 carries the other links. Links 2 and 6 are assumed to be in a plane while the rest of the links move in a parallel plane.

First, we introduce the fixed coordinate frame $\left\{x_{0}, y_{0}, z_{0}\right\}$ which is located at point $\mathrm{O}$ as shown in Fig. 2. In addition, the $z_{0}$-axis is chosen to be in line with the first actuator axis. For convenience, coordinate frame $\left\{x_{i}, y_{i}, z_{i}\right\}$ is attached to link $i(i=2 \ldots 9)$ at the revolute joint $O_{i}$ in accordance with the following rule: The $y_{i}$ - axis coincides with the largest of the link, the $x_{i}$ - axis is perpendicular to the moving plane of the link. For the link 1 , the $x_{1}$ - axis coincides with the second and third actuator axes and the $z_{1}$ - axis coincides with the $z_{0}$ - axis of the fixed frame. The configuration of the manipulator is also prescribed by three rotation angle $\theta_{1}, \theta_{2}, \theta_{3}$, in which $\theta_{1}$ represents the rotation of the link 1 around the $z_{0}$ - axis, $\theta_{2}$ and $\theta_{3}$ denote the rotation of the links 2 and 9 around the $x_{1}$ - axis respectively. The independent generalized coordinates $\boldsymbol{q}=\left[\theta_{1}, \theta_{2}, \theta_{3}\right]^{T}$ are chosen.

The position vector $\mathbf{r}_{i}$ of the center of mass $C_{i}$ in the fixed coordinate frame $\left\{x_{0}, \mathrm{y}_{0}\right.$, 
$\left.z_{0}\right\}$ is given by

$$
\boldsymbol{r}_{i}=\boldsymbol{r}_{\mathrm{i}}+\boldsymbol{A}_{i} \boldsymbol{r}_{i}^{(i)}, \quad(i=1, \ldots, 9),
$$

where $\boldsymbol{r}_{i}^{(i)}$ is position vector of $C_{i}$ in the moving coordinate frame $\left\{x_{i}, \mathrm{y}_{i}, z_{i}\right\}$ and $\boldsymbol{r}_{O i}$ is position vector of origin $O_{i}$ with respect to the fixed frame $\left\{x_{0}, \mathrm{y}_{0}, \mathrm{z}_{0}\right\}$. The rotation matrix $\boldsymbol{A}_{i}$ of the $i$-th link of the manipulator can be expressed as

$$
\begin{aligned}
& \boldsymbol{A}_{1}=\left[\begin{array}{lll}
C_{1} & -S_{1} & 0 \\
S_{1} & C_{1} & 0 \\
0 & 0 & 1
\end{array}\right] \\
& \boldsymbol{A}_{2}=\boldsymbol{A}_{4}=\boldsymbol{A}_{5}=\boldsymbol{A}_{7}=\boldsymbol{A}_{8}=\left[\begin{array}{ccc}
C_{1} & -S_{1} & 0 \\
S_{1} & C_{1} & 0 \\
0 & 0 & 1
\end{array}\right]\left[\begin{array}{ccc}
1 & 0 & 0 \\
0 & C_{2} & -S_{2} \\
0 & S_{2} & C_{2}
\end{array}\right]=\left[\begin{array}{ccc}
C_{1} & -C_{2} S_{1} & S_{1} S_{2} \\
S_{1} & C_{1} C_{2} & -C_{1} S_{2} \\
0 & S_{2} & C_{2}
\end{array}\right] \\
& \boldsymbol{A}_{9}=\boldsymbol{A}_{6}=\boldsymbol{A}_{3}=\left[\begin{array}{ccc}
C_{1} & -S_{1} & 0 \\
S_{1} & C_{1} & 0 \\
0 & 0 & 1
\end{array}\right]\left[\begin{array}{ccc}
1 & 0 & 0 \\
0 & C_{3} & -S_{3} \\
0 & S_{3} & C_{3}
\end{array}\right]=\left[\begin{array}{ccc}
C_{1} & -C_{3} S_{1} & S_{1} S_{3} \\
S_{1} & C_{1} C_{3} & -C_{1} S_{3} \\
0 & S_{3} & C_{3}
\end{array}\right]
\end{aligned}
$$

where $C_{k}$ is a shorthand notation for $\cos \theta_{k}$ and $S_{k}$ for $\sin \theta_{k}, k=1,2,3$.

Thus, position vectors of the mass centers can be derived from equations (3.1) - (3.4) and from Fig. 2 as

$$
\begin{aligned}
& r_{1}=\left[\begin{array}{lll}
0 & 0 & -d_{1}
\end{array}\right]^{T} \text {, } \\
& r_{2}=\left[\begin{array}{c}
-d_{2} C_{2} S_{1}+a_{2} C_{1} \\
d_{2} C_{2} C_{1}+a_{2} S_{1} \\
d_{2} S_{2}
\end{array}\right] \text {, } \\
& r_{3}=\left[\begin{array}{c}
-\left(-l_{4} C_{2}+d_{3} C_{3}\right) S_{1}-a_{3} C_{1} \\
\left(-l_{4} C_{2}+d_{3} C_{3}\right) C_{1}-a_{3} S_{1} \\
-l_{4} S_{2}+d_{3} S_{3}
\end{array}\right] \text {, } \\
& \boldsymbol{r}_{4}=\left[\begin{array}{c}
-\left(d_{44} C_{2}-l_{3}^{\prime} C_{3}\right) S_{1}-a_{3} C_{1} \\
\left(d_{44} C_{2}-l_{3}^{\prime} C_{3}\right) C_{1}-a_{3} S_{1} \\
d_{44} S_{2}-l_{3}^{\prime} S_{3}
\end{array}\right], \\
& r_{5}=\left[\begin{array}{c}
-\left(d_{54} C_{2}+l_{3} C_{3}\right) S_{1}-a_{3} C_{1} \\
\left(d_{54} C_{2}+l_{3} C_{3}\right) C_{1}-a_{3} S_{1} \\
d_{54} S_{2}+l_{3} S_{3}
\end{array}\right] \text {, } \\
& \boldsymbol{r}_{6}=\left[\begin{array}{c}
-\left(l_{24} C_{2}+d_{6} C_{3}\right) S_{1}+a_{2} C_{1} \\
\left(l_{24} C_{2}+d_{6} C_{3}\right) C_{1}+a_{2} S_{1} \\
l_{24} S_{2}+d_{6} S_{3}
\end{array}\right] \text {, } \\
& r_{7}=\left[\begin{array}{c}
-\left(d_{74} C_{2}-l_{5}^{\prime} C_{3}\right) S_{1}-a_{3} C_{1} \\
\left(d_{74} C_{2}+l_{5}^{\prime} C_{3}\right) C_{1}-a_{3} S_{1} \\
d_{74} S_{2}-l_{5}^{\prime} S_{3}
\end{array}\right] \text {, } \\
& \boldsymbol{r}_{8}=\left[\begin{array}{c}
-\left(d_{84} C_{2}+l_{5} C_{3}\right) S_{1}-a_{3} C_{1} \\
\left(d_{84} C_{2}+l_{5} C_{3}\right) C_{1}-a_{3} S_{1} \\
d_{84} S_{2}+l_{5} S_{3}
\end{array}\right],
\end{aligned}
$$




$$
r_{9}=\left[\begin{array}{c}
-d_{9} C_{3} S_{1}-a_{3} C_{1} \\
d_{9} C_{3} C_{1}-a_{3} S_{1} \\
d_{9} S_{3}
\end{array}\right]
$$

where $l_{24}=l_{2}-l_{4}$ and $d_{i 4}=d_{i}-l_{4}, i=4,5,7,8$.

The angular velocities of the links with respect to the link-fixed coordinate frame are given by

$$
\begin{aligned}
& \boldsymbol{\omega}_{1}^{(1)}=\left[\begin{array}{c}
0 \\
0 \\
\dot{\theta}_{1}
\end{array}\right]=\left[\begin{array}{ccc}
0 & 0 & 0 \\
0 & 0 & 0 \\
1 & 0 & 0
\end{array}\right]\left[\begin{array}{c}
\dot{\theta}_{1} \\
\dot{\theta}_{2} \\
\dot{\theta}_{3}
\end{array}\right], \\
& \omega_{2}^{(2)}=\omega_{4}^{(4)}=\omega_{5}^{(5)}=\omega_{7}^{(7)}=\omega_{8}^{(8)}=\left[\begin{array}{c}
\dot{\theta}_{2} \\
0 \\
0
\end{array}\right]+\boldsymbol{A}_{2}^{T}\left[\begin{array}{c}
0 \\
0 \\
\dot{\theta}_{1}
\end{array}\right]=\left[\begin{array}{ccc}
0 & 1 & 0 \\
S_{2} & 0 & 0 \\
C_{2} & 0 & 0
\end{array}\right]\left[\begin{array}{c}
\dot{\theta}_{1} \\
\dot{\theta}_{2} \\
\dot{\theta}_{3}
\end{array}\right], \\
& \omega_{3}^{(3)}=\omega_{6}^{(6)}=\omega_{9}^{(9)}=\left[\begin{array}{c}
\dot{\theta}_{3} \\
0 \\
0
\end{array}\right]+\boldsymbol{A}_{3}^{T}\left[\begin{array}{c}
0 \\
0 \\
\dot{\theta}_{1}
\end{array}\right]=\left[\begin{array}{ccc}
0 & 0 & 1 \\
S_{3} & 0 & 0 \\
C_{3} & 0 & 0
\end{array}\right]\left[\begin{array}{c}
\dot{\theta}_{1} \\
\dot{\theta}_{2} \\
\dot{\theta}_{3}
\end{array}\right] .
\end{aligned}
$$

Assumed that axes $x_{i}, \mathrm{y}_{i}, \mathrm{z}_{i}$ of the link-fixed coordinate frame are principal axes. The inertia matrix $I_{i}^{(i)}$ of $i$-th link about the center of mass $C_{i}$, referred to the principal axes, can be written in the form

$$
\boldsymbol{I}_{i}^{(i)}=\left[\begin{array}{ccc}
\boldsymbol{I}_{x i} & 0 & 0 \\
0 & \boldsymbol{I}_{y i} & 0 \\
0 & 0 & \boldsymbol{I}_{z i}
\end{array}\right], \quad i=1,2, \ldots, 9
$$

By using the expressions from (3.5) to (3.13) we obtain

$$
\sum_{i=1}^{9} m_{i} \boldsymbol{J}_{T i}(\boldsymbol{q})=\left[\begin{array}{ccc}
f_{1} S_{1}+f_{2} C_{1} C_{2}+f_{3} C_{1} C_{3} & -f_{2} S_{1} S_{2} & -f_{3} S_{1} S_{3} \\
-f_{1} C_{1}+f_{2} S_{1} C_{2}+f_{3} S_{1} C_{3} & f_{2} S_{2} C_{1} & f_{3} S_{3} C_{1} \\
0 & -f_{2} C_{2} & -f_{3} C_{3}
\end{array}\right]
$$

where

$$
\begin{aligned}
& f_{1}=-\left(m_{2}+m_{6}\right) a_{2}+\left(m_{3}+m_{4}+m_{5}+m_{7}+m_{8}+m_{9}\right) a_{3} \\
& f_{2}=-m_{2} d_{2}-\sum_{i=4}^{8} m_{i} d_{i}+l_{4} \sum_{i=3}^{8} m_{i}+m_{6}\left(d_{6}-l_{2}\right), \\
& f_{3}=-m_{3} d_{3}+m_{4} l_{3}^{\prime}-m_{5} l_{3}-m_{6} d_{6}+m_{7} l_{5}^{\prime}-m_{8} l_{5}-m_{9} d_{9} .
\end{aligned}
$$

Expression (3.18) contains three equations for shaking force balancing of the manipulator. Finally, by using expressions from (3.2) to (3.17) we get

$$
\sum_{i=1}^{g}\left[\boldsymbol{A}_{i} \boldsymbol{I}_{i}^{(i)} \boldsymbol{J}_{R i}^{(i)}(\boldsymbol{q})+m_{i} \tilde{\boldsymbol{r}}_{i} \boldsymbol{J}_{T i}(\boldsymbol{q})\right]=\left[\begin{array}{lll}
h_{11} & h_{12} & h_{13} \\
h_{21} & h_{22} & h_{23} \\
h_{31} & h_{32} & h_{33}
\end{array}\right]
$$

with the following elements

$$
h_{11}=f_{4} S_{2} C_{1}+f_{5} S_{3} C_{1}+f_{6}\left(S_{1} S_{3} C_{2}+S_{1} S_{2} C_{3}\right)+f_{8} S_{1} S_{2} C_{2}+f_{9} S_{1} S_{3} C_{3},
$$




$$
\begin{aligned}
& h_{12}=-f_{4} S_{1} C_{2}+f_{6}\left(S_{2} S_{3} C_{1}+C_{1} C_{2} C_{3}\right)+f_{10} C_{1}, \\
& h_{13}=-f_{5} S_{1} C_{3}+f_{6}\left(S_{2} S_{3} C_{1}+C_{1} C_{2} C_{3}\right)+f_{11} C_{1}, \\
& h_{21}=f_{4} S_{1} S_{2}+f_{5} S_{1} S_{3}-f_{6}\left(S_{2} C_{1} C_{3}+S_{3} C_{1} C_{2}\right)-f_{8} S_{2} C_{1} C_{2}-f_{9} S_{3} C_{1} C_{3}, \\
& h_{22}=f_{4} C_{1} C_{2}+f_{6}\left(S_{1} S_{2} S_{3}+S_{1} C_{2} C_{3}\right)+f_{10} S_{1}, \\
& h_{23}=f_{5} C_{1} C_{3}+f_{6}\left(S_{1} S_{2} S_{3}+S_{1} C_{2} C_{3}\right)+f_{11} S_{1}, \\
& h_{31}=2 f_{6} C_{2} C_{3}+f_{8} C_{2}^{2}+f_{9} C_{3}^{2}+f_{7}, \\
& h_{32}=f_{4} S_{2}, \\
& h_{33}=f_{5} S_{3},
\end{aligned}
$$

where

$$
\begin{aligned}
f_{4}= & -m_{2} a_{2} d_{2}-m_{3} a_{3} l_{4}+m_{4} a_{3} d_{44}+m_{5} a_{3} d_{54}-m_{6} a_{2} l_{24}+m_{7} a_{3} d_{74}+m_{8} a_{3} d_{84}, \\
f_{5}= & \left(m_{3} d_{3}-m_{4} l_{3}^{\prime}+m_{5} l_{3}-m_{7} l_{5}^{\prime}+m_{8} l_{5}+m_{9} d_{9}\right) a_{3}-m_{6} a_{2} d_{6}, \\
f_{6}= & -m_{3} l_{4} d_{3}-\dot{m}_{4} l_{3}^{\prime} d_{44}+m_{5} l_{3} d_{54}+m_{6} l_{24} d_{6}-m_{7} l_{5}^{\prime} d_{74}+m_{8} l_{5} d_{84}, \\
f_{7}= & \left(m_{2}+m_{6}\right) a_{2}^{2}+\left(m_{3}+m_{4}+m_{5}+m_{7}+m_{8}+m_{9}\right) a_{3}^{2}+\sum_{i=2}^{9} I_{y i}+I_{z 1}, \\
f_{8}= & m_{2} d_{2}^{2}+m_{3} l_{4}^{2}+m_{4} d_{44}^{2}+m_{5} d_{54}^{2}+m_{6} l_{24}^{2}+m_{7} d_{74}^{2}+m_{8} d_{84}^{2} \\
& +I_{z 2}+I_{z 4}+I_{z 5}+I_{z 7}+I_{z 8}-I_{y 2}-I_{y 4}-I_{y 5}-I_{y 7}-I_{y 8} \\
f_{9}= & m_{3} d_{3}^{2}+m_{4} l_{3}^{2}+m_{5} l_{3}^{2}+m_{6} d_{6}^{2}+m_{7} l_{5}^{2}+m_{8} l_{5}^{2}+m_{9} d_{9}^{2} \\
& +I_{z 3}+I_{z 6}+I_{z 9}-I_{y 3}-I_{y 6}-I_{y 9} \\
f_{10}= & m_{2} d_{2}^{2}+m_{3} l_{4}^{2}+m_{4} d_{44}^{2}+m_{5} d_{54}^{2}+m_{6} l_{24}^{2}+m_{7} d_{74}^{2}+m_{8} d_{84}^{2}, \\
& +I_{x 2}+I_{x 4}+I_{x 5}+I_{x 7}+I_{x 8} \\
f_{11}= & m_{3} d_{3}^{2}+m_{4} l_{3}^{\prime 2}+m_{5} l_{3}^{2}+m_{6} d_{6}^{2}+m_{7} l_{5}^{2}+m_{8} l_{5}^{2}+m_{9} d_{9}^{2}+I_{x 3}+I_{x 6}+I_{x 9} .
\end{aligned}
$$

Expression (3.22) contains eight equations for shaking moment balancing of the manipulator.

\section{DISCUSSION AND CONCLUSIONS}

The manipulator is statically balanced if expressions of $f_{1}, f_{2}, f_{3}$ in $(3.19),(3.20)$ and (3.21) vanish, this yields the following conditions

$$
\begin{aligned}
& -\left(m_{2}+m_{6}\right) a_{2}+\left(m_{3}+m_{4}+m_{5}+m_{7}+m_{8}+m_{9}\right) a_{3}=0 \\
& -m_{2} d_{2}-\sum_{i=4}^{8} m_{i} d_{i}+l_{4} \sum_{i=3}^{8} m_{i}+m_{6}\left(d_{6}-l_{2}\right)=0 \\
& -m_{3} d_{3}+m_{4} l_{3}^{\prime}-m_{5} l_{3}-m_{6} d_{6}+m_{7} l_{5}^{\prime}-m_{8} l_{5}-m_{9} d_{9}=0
\end{aligned}
$$

Conditions (4.1) can be satisfied by internal mass redistribution or using counterweights mounted on the links. These conditions may give simple design guidelines for shaking force balancing of the manipulator.

Furthermore, the manipulator is completely dynamically balanced if the following expressions vanish

$$
f_{k}=0, \quad k=4,5, \ldots, 11 \text {. }
$$


Note that these conditions can not be completely satisfied in practice. For example, the values of $f_{7}, f_{10}$ and $f_{11}$ are not equal to zero in any case. However, the static balanced manipulator can be partially dynamically balanced if some conditions in (4.2) are satisfied, for example $f_{4}=f_{5}=f_{6}=0$. Another solution for this problem is the minimization of the shaking moment, this yields a set of optimizing values for geometrical and inertia parameters of the links

$$
f_{k} \rightarrow \min , \quad k=4,5, \ldots, 11 .
$$

The research for shaking moment minimization of spatial multibody systems will be done in the future.

Acknowledgment. This paper was completed with the financial support of the Vietnam Basic Research Program in Natural Science.

\section{REFERENCES}

1. Nguyen Van Khang, Über den Massenausgleich in Mehrkörpersystemen. Technische Mechanik, Band 14, Heft 3/4 (1994) 231-238.

2. T. M. Abdel-Rahman, M. A. Elbestawi, Synthesis and Dynamics of Statically Balanced Direct-drive Manipulators with Decoupled Inertia Tensors. Mechanism and Machine Theory 26 (1991) 389-402.

3. M. Kolarski, M. Vukobratovic, B. Borovac, Dynamic Analysis of Balanced Robot Mechanisms. Mechanism and Machine Theory 29 (1994) 427-454.

4. Y. Yue-Qing, Complete Shaking Force and Moment Balancing of SpatiaI Irregular Force Transmission Mechanisms Using Additional Link. Mechanism and Machine Theory 23 (1988) 279-285.

5. V. Arakelian, M. R. Smith, Shaking Moment Minimization of Fully Force-Balanced Linkages. Proc. of the $11^{\text {th }}$ World Congress in Mechanism and Machine Science, Tianjin, China, 2004.

6. I. S. Kochev, General Method for Active Balancing of Combined Shaking Moment and Torque Fluctuations in Planar Linkages. Mechanism and Machine Theory 25 (1990) 679-687.

7. H. Dresig, I. I. Vulfson, Dynamik der Mechanismen, Springer Verlag, Wien, 1989.

8. W. Schiehlen, Technische Dynamik, B.G. Teubner, Stuttgart, 1986.

Received July 25, 2005

\section{CÂN BẰNG LỰC QUÁN TÍNH VÀ MÔ MEN LỰC QỬN TÍNH CỦA HỆ NHIỀU VẬT KHÔNG GIAN CÓ CẤU TRƯC CHUỖI ĐộNG Hở}

Bài báo này đề cập tới một giải pháp cho vấn đề cân bẵng hoàn toàn lực quán tính và mô men lực quán tính của hệ nhiều vật không gian có cấu trúc chuỗi động hở. Trước hết, các điều kiện tổng quát đề cân bằng lực quán tính và mô men lực quán tính của hệ nhiều vật không gian được thiết lập. Các điều kiện này bao gồm các công thức dẫn đến sự triệt tiêu hoàn toàn véc tơ chính và mô men chính của hệ lực quán tính gây ra bởi các vật thuộc hệ. Các hệ thức cân bằng lực quán tính và mô men lực quán tính của một tay máy chín khâu được trình bày trong một thí dụ áp dụng. 